\title{
Comercio e inversión entre México y Chile
}

$\mathrm{L}$ a política comercial de Chile está orientada a una inserción internacional basada en la apertura comercial, la cual se desarrolla desde tres ángulos que resultan complementarios para los fines que se persiguen: la apertura unilateral, que se viene aplicando desde los años setenta, las negociaciones comerciales multilaterales y la apertura negociada a nivel bilateral.

La apertura unilateral chilena tuvo como fin lograr el crecimiento de las exportaciones tradicionales y no tradicionales, además de estimular una mayor diversificación en productos y mercados de destino. Con ese fin se redujo el arancel general que pagan las importaciones del 15 al 11 por ciento entre 1991 y 1998. Además Chile se comprometió a reducir un punto porcentual a partir de1999, hasta llegar a 6 por ciento en 2003.

Al igual que otras economías que se adhieren al sistema multilateral de la Organización Mundial del Comercio (OMC), Chile se comprometió en la Ronda Uruguay a ajustar el nivel arancelario a sus intereses: la tarifa máxima acordada fue de 25 por ciento antes, el país mantenía un arancel de 35 por ciento-, exceptuando a los bienes que se encuentran bajo el sistema de bandas de precios: trigo, harina de trigo, azúcar y aceites, productos que junto a los lácteos quedaron con un gravamen máximo de 31.5 por ciento.

Una muestra clara de los esfuerzos que realiza Chile para abrir sus mercados al exterior y promover sus productos en el extranjero son los Acuerdos de Complementación Económica (ACE) y los Tratados de Libre Comercio (TLC) que ha celebrado con diferentes economías. Por ejemplo: los ACE celebrados con el Mercado Común del Sur (MERCOSUR), entró en vigencia en 1998; el de Chile-Perú en julio de 1988; el

* Investigadora del Departamento de Estudios del Pacífico de la Universidad de Guadalajara. de Chile-Ecuador fue firmado en 1994; ChileCanadá (TLC) el 5 diciembre de 1996, así como el TLC Chile-Centroamérica (el 18 de octubre de 1999); además, Chile firmó un Acuerdo de Promoción y Protección de Inversiones (APPIs) con Costa Rica (11 de julio de 1996); con el Salvador, Nicaragua y Guatemala suscribió convenios de esta misma naturaleza (8 de noviembre de 1996). En 1998 Chile firmó un TLC con México (este tratado sustituye al ACE de 1991 y que estuvo vigente por siete años). Además, Chile mantiene negociaciones para la posible firma de acuerdos o tratados de libre comercio con Corea, Estados Unidos y la Unión Europea.

En este trabajo se brinda un panorama general de la situación que guarda el comercio de Chile con sus principales socios comerciales, destacando la relación entre México y Chile.

\section{El comercio de Chile}

Las políticas comerciales que se han adoptado en Chile están orientadas al apoyo de los sectores que no son tradicionalmente exportadores e incluso aquellos que son potencialmente exportadores; aunque no se reconocen como subsidios a la exportación, existen actividades relacionadas con esta actividad que dependen en gran medida de atención específica, lo que podría constituirse indirectamente como un subsidio a la exportación.

Por otro lado, el ministro de asuntos exteriores de Chile promueve exportaciones a otros países; la oficina de promoción de exportaciones hace promoción en productos específicos, se les proporciona asistencia financiera directa a las empresas participantes, existen programas de simplificación a exportaciones no tradicionales y se otorgan descuentos al pago por la importación de componentes. 
Otro de los apoyos importantes que reciben las empresas domésticas compradoras de equipo de capital es el préstamo hasta del 73 por ciento de la cantidad que pagará por los bienes de capital que ellos han importado; si los bienes de capital son usados en la producción final de los productos de exportación no necesita pagar el interés del préstamo ni el pago del monto del préstamo, o bien se les permite a todos los exportadores importar, por un plazo de más de siete años, equipo de capital.

De 1996 a 2000, las exportaciones chilenas experimentaron un crecimiento de 18.2 por ciento promedio anual (de 15 353 millones de dólares a 18 158 millones de dólares). Por su parte en el año 2000 las importaciones totalizaron 16 731 millones de dólares mientras en 1996 fueron de 16499 millones de dólares, por lo que el crecimiento de las importaciones fue sólo de 1 por ciento entre $1996 \mathrm{y}$ 2000. El principal destino de las exportaciones chilenas es Estados Unidos, seguido por Asia y Europa (cuadro 1). 17.3 por ciento que Chile exporta al exterior lo hace a Estados Unidos, 13.8 por ciento a Japón y 5.8 por ciento a Inglaterra. Las exportaciones del sector agropecuario chileno se han constituido en la más importante fuente de exportación a Estados Unidos, al cual exporta productos provenientes en su mayoría del sector agropecuario (principalmente productos frutícolas y mineros), mientras que hacia los otros mercados las exportaciones del ramo de $\mathrm{recurs}$ o $\mathrm{s}$ naturales están concentradas únicamente en la minería, en tanto que al mercado sudam e ricano concentra una mayor proporción de exportaciones de $\mathrm{b}$ i e $\mathrm{n}$ e $\mathrm{s}$ $\mathrm{m}$ a $\mathrm{nufac}$ turados $(36.9$

por ciento en el año 2000).

Por el contrario, la participación de las exportaciones de Estados Unidos en América Latina y el Caribe es menor en el volumen que se dirige a Chile (ocupa el lugar número treinta y dos). Las importaciones de Estados Unidos provenientes de Chile en 2001 fueron de 3.6 miles de millones de dólares, lo que refleja una disminución de 8.7 por ciento respecto al año anterior. incluso mantiene superávit comercial por largos períodos fue de 3.8 por ciento y al nordeste asiático de solo el 1.0 por ciento en los mismos productos.

La apertura comercial que ha implementado Chile se combina con la suscripción de las APPIs y, recientemente, con convenios que eviten la doble tributación. En 


\section{Cuadro 2}

Chile: principales mercados de destino de las exportaciones, 1998-2000 (millones de dólares y porcentajes)

\begin{tabular}{lrrr|rr|r}
\hline Mercado & & & & \multicolumn{2}{c|}{ Participación } & \multicolumn{2}{c}{ Variación } \\
\hline 1 Estados Unidos & $2,610.0$ & $3,087.5$ & $3,183.7$ & 19.4 & 17.3 & $\mathbf{1 9 9 9 / 2 0 0 0}$ (\%) \\
2 Japón & $1,956.4$ & $2,276.3$ & $2,548.5$ & 14.3 & 13.8 & 3.1 \\
3 Inglaterra & $1,161.1$ & $1,085.2$ & $1,075.9$ & 6.8 & 5.8 & 12.0 \\
4 Brasil & 778.9 & 687.6 & 961.6 & 4.3 & 5.2 & -0.9 \\
5 China & 459.7 & 359.1 & 907.2 & 2.3 & 4.9 & 39.8 \\
6 Italia & 668.5 & 636.7 & 825.5 & 4.0 & 4.5 & 152.6 \\
7 México & $\mathbf{4 8 8 . 4}$ & $\mathbf{6 2 2 . 8}$ & $\mathbf{8 1 8 . 1}$ & $\mathbf{3 . 9}$ & $\mathbf{4 . 4}$ & 29.7 \\
8 Corea & 384.7 & 683.7 & 806.3 & 4.3 & 4.4 & $\mathbf{3 1 . 4}$ \\
9 Argentina & 735.1 & 727.4 & 638.6 & 4.6 & 3.5 & 17.9 \\
10 Francia & 443.8 & 495.4 & 633.1 & 3.1 & 3.4 & -12.2 \\
11 Taiwan & 524.5 & 509.6 & 610.7 & 3.2 & 3.3 & 27.8 \\
12 Alemania & 537.9 & 555.6 & 457.8 & 3.5 & 2.5 & 19.8 \\
13 Holanda & 432.7 & 511.1 & 452.7 & 3.2 & 2.5 & -17.6 \\
14 Perú & 352.9 & 354.1 & 439.2 & 2.2 & 2.4 & -11.4 \\
15 España & 274.9 & 328.1 & 386.2 & 2.1 & 2.1 & 24.0 \\
16 Bélgica & 345.1 & 280.2 & 375.1 & 1.8 & 2.0 & 17.7 \\
17 Canadá & 143.4 & 173.5 & 243.7 & 1.1 & 1.3 & 33.9 \\
18 Colombia & 211.4 & 206.0 & 236.8 & 1.3 & 1.3 & 40.5 \\
19 Venezuela & 176.8 & 196.4 & 228.6 & 1.2 & 1.2 & 15.0 \\
20 Bolivia & 249.6 & 193.3 & 164.5 & 1.2 & 0.9 & 16.4 \\
& & & & & & -14.9 \\
Subtotal & $12,936.1$ & $13,969.4$ & $15,994.0$ & 87.8 & 86.8 & 14.5 \\
Total & $14,753.9$ & $15,914.6$ & $18,425.0$ & 100.0 & 100.0 & 15.8 \\
\hline
\end{tabular}

Fuente: gobierno de Chile, Ministerio de Relaciones Económicas Internacionales, Dirección de Estudios, diciembre de 2000.

los últimos años, a la apertura de bienes e inversiones se suma también la liberalización de los servicios (transporte, energía, telecomunicaciones y otros servicios).

\section{Relación comercial México-Chile}

El comercio entre México y Chile se ha mantenido en crecimiento casi continuo desde principios de los años ochenta, incluso México mantuvo superávit comercial por largos períodos como sucedió de 1983 a 1995 (con excepción de 1993 que se registró disminución en las exportaciones mexicanas), de 1996 a 1998 se mantiene con ligeros altibajos. Sin embargo, al entrar en vigor el TLC México-Chile (en agosto de 1999) incrementó el volumen de las exportaciones e importaciones mexicanas (gráfica 1). Con este resultado en el comercio, se cumple con uno de los objetivos propuestos en la firma del TLC entre los dos países, el cual fue de incrementar los flujos comerciales, como una consecuencia de la eliminación o en su caso la disminución de las barreras arancelarias entre estos países.

México tiene 89 productos con aranceles preferenciales, de los cuales 26 cuentan con reducciones entre 28 y 70 por ciento, aunque quedaron fuera de la negociación algunos productos como lácteos, la cebada, el trigo y las manzanas frescas. Chile incluye en la lista de productos exceptuados 96 de los cuales 42 
disminuyeron su arancel entre 12 y 71 por ciento. Los aranceles que afectan a éstos comenzaron a disminuir en enero de 1999, proceso que concluirá en el 2006. Además de complementar la apertura lograda a través del ACE, el TLC considera un régimen especial para el sector automotor, que será desgravado totalmente en enero de 1996. Este convenio establece además un sistema eficiente de solución de controversias que permite resolver en forma clara y transparente los problemas que puedan surgir entre las partes en el marco del comercio bilateral. Dentro de las materias que abarca el TLC se encuentran las disposiciones sobre instituciones, inversiones y servicios, transporte aéreo, telecomunicaciones, acceso a mercados, reglas de origen y salvaguardas.

La relación comercial México-Chile está aún a niveles bajos, pues apenas México se ubica en el séptimo lugar en importancia como destino de las exportaciones chilenas después de Estados Unidos, Japón, Inglaterra, Brasil, China e Italia (cuadro 2).

\section{Inversión extranjera directa}

A través del Comité de Inversión Extranjera (FIC por sus siglas en inglés) del ministerio de economía ingresa IED al país bajo el Decreto Ley (DL) 600, con los términos y condiciones que se han establecido. Los beneficios pueden ser repatriados inmediatamente, pero ningún capital original puede ser repatriado por un año. El FIC señala condiciones diferentes para cada inversor. En el caso de los minerales, el período es por ocho años, el cual puede extenderse hasta doce años si la empresa tiene como actividad la exploración. En todas las demás áreas el período es de tres años. En el caso de la inversión industrial o de los proyectos extractivos (excluyendo minas) que ascienden hasta 50 millones de dólares, el término puede ser extendido hasta ocho años dependiendo de la naturaleza del proyecto. La aprobación de FIC se hace en los siguientes tipos de inversión: los que exceden un valor total a 5 millones, los que se relacionan con sectores o actividades que normalmente son desarrolladas por el gobierno o que tienen como finalidad dar un servicio público, y los proyectos realizados por gobiernos o entidades públicas foráneas.

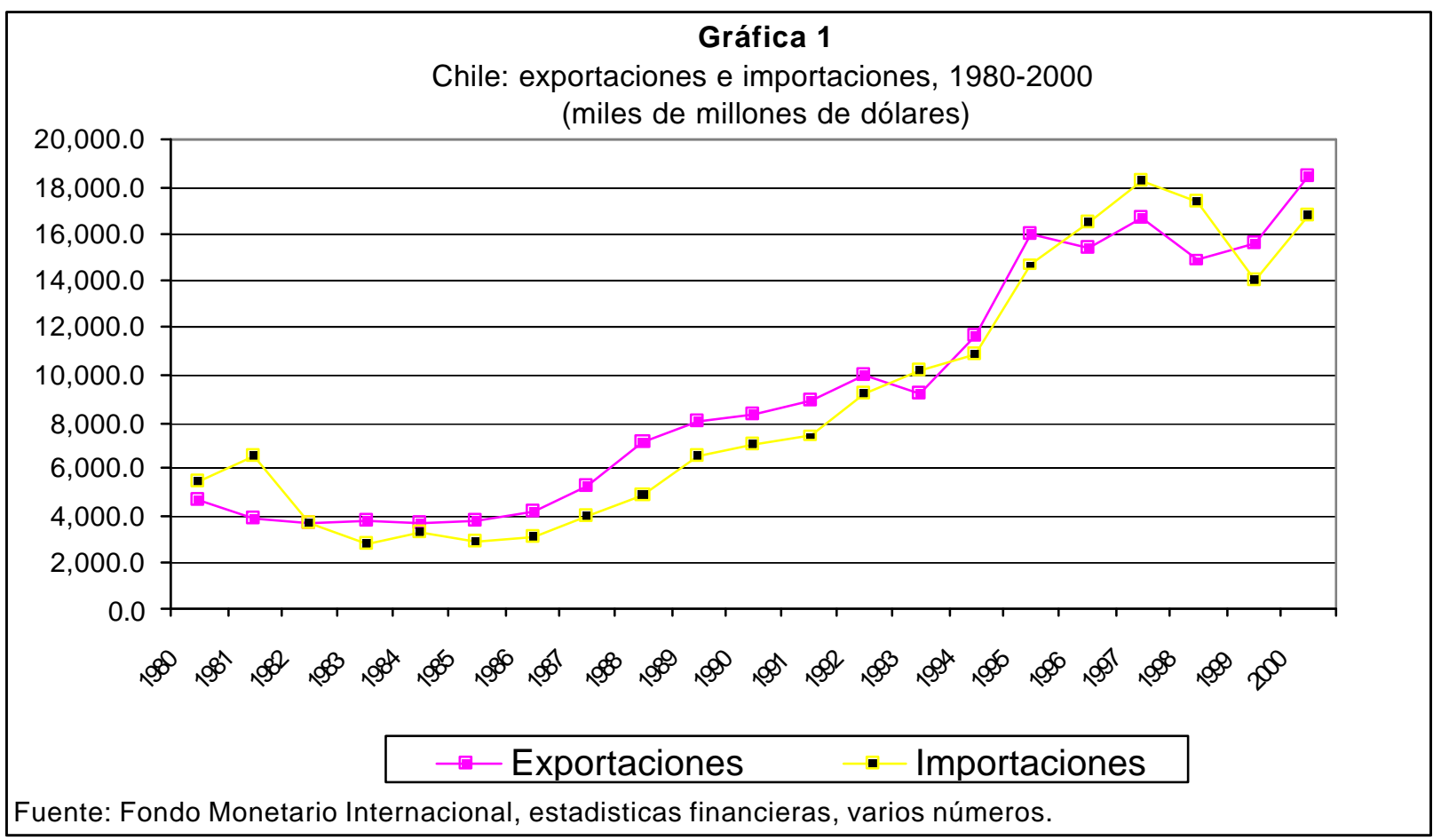




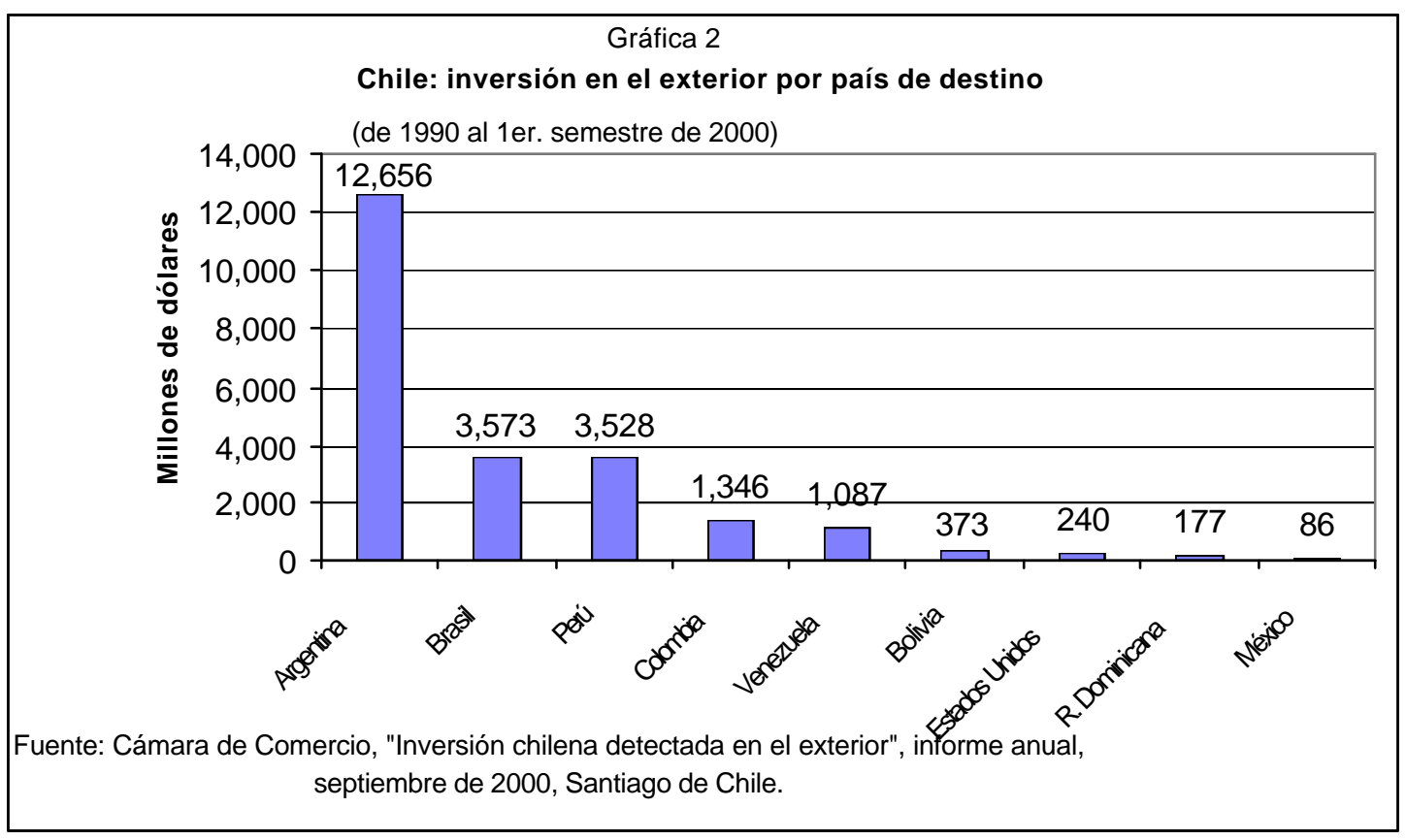

El total de monto acumulado de IED que Chile tiene en el exterior asciende a 23,333 millones de dólares, cuyo marcado crecimiento se observa desde principios de los noventa cuando se agudiza la firma de convenios y tratados de libre comercio. De 1993 a 1994 experimenta un crecimiento de 276.7 por ciento en los flujos de inversión y de 1995 a 1996 crece 53 por ciento, siendo en este último año cuando alcanza el nivel máximo del período que comprende 1990-1999 (6, 368 millones de dólares). No obstante después de 1996 experimenta una disminución considerable hasta llegar a 1417 millones de dólares en 1999 (gráfica 2). La inversión de origen chileno en México y Estados Unidos, tiene menor relevancia, ya que estos ocupan el noveno y séptimo lugar respectivamente. Argentina se ha mantenido año tras año como el primer país de destino de la inversión acumulada de Chile; entre 1990 y primer semestre de 2000 ésta ascendió a 12656 millones de dólares, equivalentes a 53.0 por ciento del total de la inversión chilena en el extranjero. Brasil y Perú representan 15.0 por ciento y 14.8 por ciento respectivamente, con una importante desaceleración del segundo a partir del último trimestre de 1999 ( gráfica 2 ).
Mientras las inversiones en Argentina y Perú están distribuidas de manera más homogénea entre energía, industria y comercio, en el caso brasileño se concentran básicamente en energía e industria, al igual que sus inversiones totales que también se concentran en esos sectores sumando los dos el 74.6 por ciento.

El stock de IED en Chile originario de Estados Unidos en 2000 fue de 10.8 miles de millones de dólares, un incremento del 7.3 por ciento respecto a 1999. La IED de Estados Unidos en Chile está concentrada principalmente en finanzas, manufacturas y el sector bancario.

Para estimular la afluencia de capitales en Chile, se decidió eliminar el requisito de permanencia mínima de un año que se aplicaba a las inversiones externas. Otras importantes medidas adoptadas fueron, la abolición del impuesto sobre las ganancias de capital a no residentes y la flexibilización del porcentaje de endeudamiento ingreso como Inversión Extranjera Directa (IED). Así, subsidiarias de Nestlé, Unilever, General Motors, Coca Cola y Procter \& Gamble siguen ocupando lugares privilegiados entre las mayores empresas de 
capital extranjero en la economía chilena con creciente especialización de la producción. Las empresas extranjeras fueron decisivas en el éxito de la primera fase de desarrollo económico chileno basado en las exportaciones, para luego progresivamente retirarse de aquellas actividades más sensibles a los ciclos de precios internacionales. No obstante, en lo que respecta a la participación inicial de las empresas transnacionales en el sector servicios fue poco significativa, ya que los grupos locales mantuvieron el liderazgo tras las privatizaciones a inicios de los años ochenta. Constituye la principal excepción la telefonía básica fija. Sin embargo, ante el buen desempeño de tales sectores, las compañías foráneas buscaron controlar las empresas, desencadenando una ola sin precedentes de fusiones y adquisiciones. En síntesis, en los últimos años, las empresas extranjeras han manifestado dos estrategias básicas en la economía de Chile: en primer lugar, la búsqueda de materias primas para la exportación. Los abundantes recursos naturales que posee Chile lo han convertido en destino de las principales empresas transnacionales del sector minero y de algunas actividades vinculadas a la agricultura y silvicultura. En los últimos años, el interés de la inversión extranjera ha comenzado a trasladarse de los productos básicos basados en recursos naturales (celulosa y papel, harina de pescado) a productos menos estandarizados con certificación de origen (vinos finos y salmones). En segundo lugar, el acceso a mercados locales y regionales en sectores de servicios; es el caso de las telecomunicaciones, los servicios financieros y más recientemente la generación y distribución de energía eléctrica y los servicios sanitarios y de agua potable. El prematuro liderazgo regional de algunos grupos locales despertó el interés de las empresas transnacionales que están intentando posicionarse internacionalmente como grupos globales de servicios.

Las primeras negociaciones en el ámbito de la liberalización del sector servicios se llevaron a cabo en el inicio de los años noventa, culminando con el Acuerdo General sobre Comercio de Servicios (AGCS, vigente desde el
1 de enero de 1995). Chile es miembro fundador de ese convenio. Entre los principales objetivos de Chile a nivel internacional destaca el establecimiento de reglas claras para el intercambio de servicios e inversiones, con vistas a crear un mercado más amplio para estas actividades en otros países. En este sentido se busca abrir progresivamente los mercados para los prestadores de servicios así como fortalecer los procesos de integración que contribuyan a la expansión del comercio y favorezcan la generación de alianzas estratégicas con empresas de otros países, a fin de penetrar en nuevos mercados.

En las telecomunicaciones destaca la no discriminación entre inversores nacionales y extranjeros, no existiendo un límite para su participación en empresas del país. El marco regulatorio chileno entrega un amplio margen de acción para que el mercado se desarrolle, y sólo vigila posibles abusos de posición dominante de mercado; esto se logra mediante un proceso llamado "decreto tarifario" que establece, por un período de cinco años, las cantidades máximas a cobrar por servicios de larga distancia, telefonía local o internet. En transporte aéreo de carga y pasajeros, hay libertad para ofrecer este servicio, pero a nivel internacional existe una excepción vinculada a la reciprocidad establecida en los acuerdos bilaterales de manera que las empresas extranjeras puedan operar en este ámbito siempre que sus Estados otorguen preferencias similares a las compañías de la otra parte signataria. Considerando el dinamismo del comercio exterior de Chile, la vía aérea se ha transformado en una importante fuente de transporte para los productos que ingresan y salen del país. Las empresas nacionales y extranjeras, para ofrecer servicio aéreo, sólo se rigen por la Ley de aviación comercial. En los servicios financieros, la privatización y la apertura a la inversión internacional han sido la base de los cambios que ha sufrido el sector financiero en los últimos años. A nivel multilateral, aún no se alcanza un acuerdo sobre la materia; sin embargo, en Chile la legislación no discrimina entre el trato a nacionales y a extranjeros, buscando facilitar un flujo permanente de capitales. La regulación y 
Cuadro 3

Chile:exportaciones según acuerdos con programas de liberalización

1996-2001

(millones de dólares)

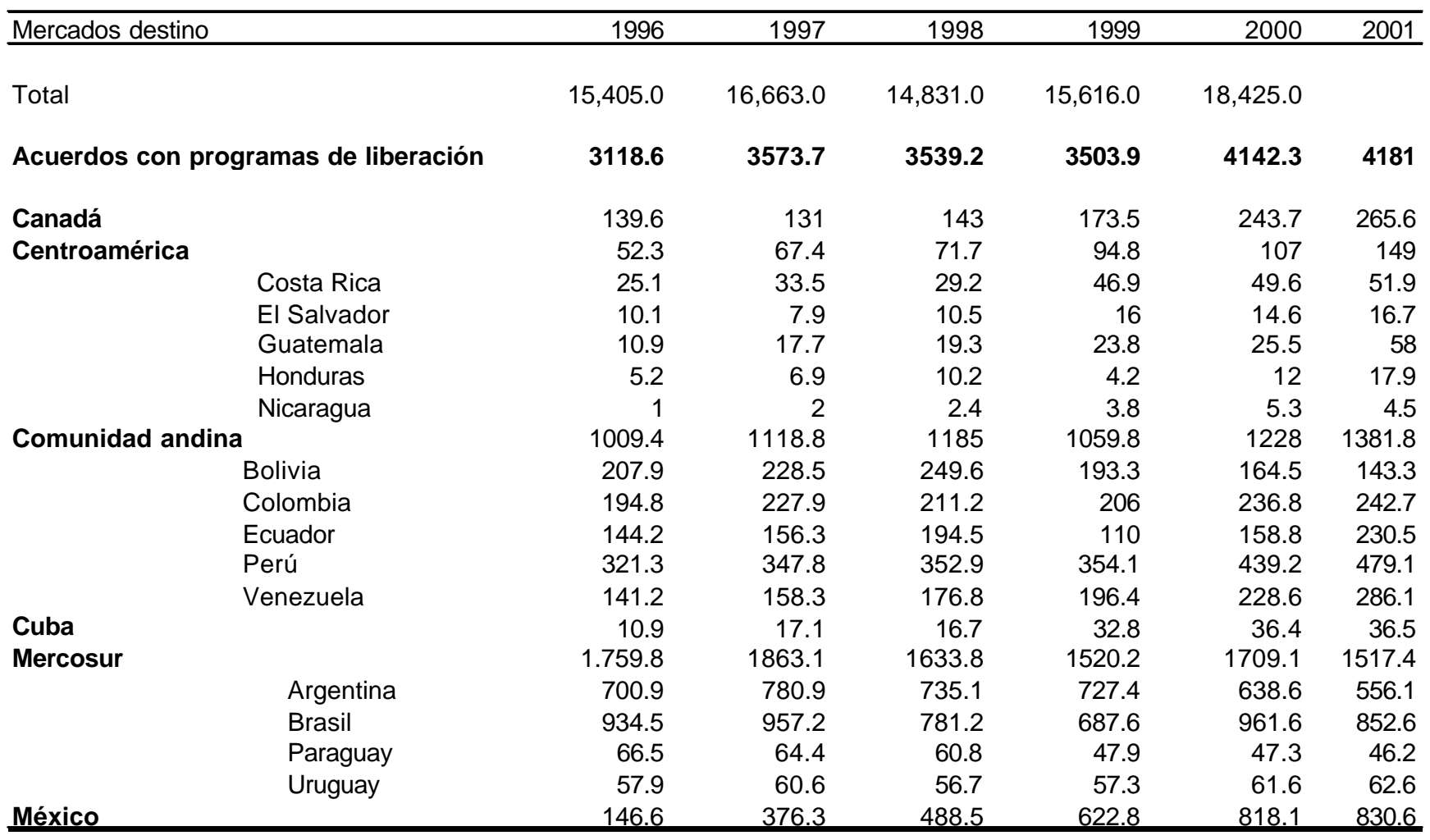

Fuente: gobierno de Chile, Ministerio de Relaciones Económicas Internacionales, Dirección de Estudios, diciembre de 2000.

supervisión del sector financiero está a cargo de la Superintendencia de Bancos Institucionales Financieros (SBIF), dependiente del Ministerio de Hacienda, y del Banco Central de Chile. Para adquirir acciones en bancos y filiales chilenas se debe contar con personalidad jurídica en este país.

\section{Inversión de Chile en México}

México cuenta con 207 empresas con inversión proveniente de Chile, lo cual representa 0.9 por ciento del total de empresas con IED establecidas en México (23 110).

Del grupo de los países de la Asociación Latinoamericana de Integración (ALADI), Chile ocupa la segunda posición como país inversor en México, con 74.1 millones de dólares, monto que equivale a 22 por ciento del monto de ese grupo.

Las empresas con capital chileno participan mayoritariamente en el sector servicios, representando 41.5 por ciento del total de la IED de ese país ubicada en México( la cual se concentra en servicios de instituciones crediticias, bancarias, de seguros y fianzas, de alquiler de equipo de maquinaria y equipo, servicios médicos y servicios profesionales entre otros), en comercio 33.8 por ciento (comercio de alimentos bebidas y tabaco, productos no alimenticios), en la industria 12.6 por ciento (fabricación de sustancias químicas, de estructuras metálicas, imprentas, editoriales, del calzado, cuero y pieles). En el sector 
agropecuario (silvicultura y pesca) 3.9 por ciento, construcción, edificación e instalaciones 4.8 por ciento; en otros sectores menos representativos se ubica el resto.

\section{Comentarios finales}

No obstante los acuerdos celebrados por Chile con otros países bajo programa de liberalización comercial no ha habido cambios significativos; de 1996 a 2000 tan solo se incrementaron las exportaciones 2.2 ( de 20.3 se incrementó a 22.5 por ciento) por ciento (cuadro 3), en tanto que en las importaciones, bajo estas circunstancias, se incrementaron 8.2 por ciento en el mismo período ( de 30.4 incrementó a 38.5 por ciento). Sin embargo, Chile busca las condiciones de apertura de comercio e inversión con la finalidad de no quedarse fuera de los beneficios del libre comercio. Aun cuando las condiciones no se han dado para una mayor diversificación del comercio, este país está a la espera de las oportunidades que vayan llegando con el avance de la eliminación de las barreras comerciales que cada día van desapareciendo con los acuerdos y tratados comerciales, ya sean bilaterales o multilaterales, muy de moda actualmente.

\section{Fuentes}

Gobierno de Chile, "Inversión extranjera directa y estrategias empresariales, la inversión extranjera directa en América Latina y el Caribe", 2000.

INEGI, Banco de Información Económica, 2002.

Gobierno de Chile, Dirección de Estudios-DIRECON, mayo, 2001.

Cámara de Comercio de Santiago, Departamento de Estudios, "Inversión chilena detectada en el exterior", informe, septiembre, 2000. 